\title{
Remarques sur le premier cas du théorème de Fermat sur les corps de nombres
}

\begin{abstract}
Alain Kraus
Abstract. The first case of Fermat's Last Theorem for a prime exponent $p$ can sometimes be proved using the existence of local obstructions. In 1823, Sophie Germain has obtained an important result in this direction by establishing that, if $2 p+1$ is a prime number, the first case of Fermat's Last Theorem is true for $p$. In this paper, we investigate such obstructions over number fields. We obtain analogous results on Sophie Germain type criteria, for imaginary quadratic fields. Furthermore, extending a well known statement over $\mathbb{Q}$, we give an easily testable condition which allows occasionally to prove the first case of Fermat's Last Theorem over number fields for a prime number $p \equiv 2$ mod. 3 .
\end{abstract}

\section{AMS Mathematics Subject Classification : 11D41}

Keywords : First Case of Fermat's Last Theorem - Number fields.

\section{INTRODUCTION}

Soient $K$ un corps de nombres et $p$ un nombre premier impair. On dit que le premier cas du théorème de Fermat est vrai sur $K$ pour l'exposant $p$, s'il n'existe pas d'éléments $x, y, z$ dans l'anneau d'entiers $O_{K}$ de $K$ tels que

$$
x^{p}+y^{p}+z^{p}=0 \quad \text { et } \quad(x y z) O_{K}+p O_{K}=O_{K}
$$

En 1994, A. Wiles a démontré qu'il en est ainsi pour le corps $\mathbb{Q}$, indépendamment du fait que $x y z$ soit premier avec $p([\mathrm{Wi}])$. En 2004 , ce résultat a été étendu au corps $\mathbb{Q}(\sqrt{2})$ par F. Jarvis et P. Meekin ([Ja-Me]). Récemment, N. Freitas et S. Siksek ont accompli des progrès importants concernant l'équation de Fermat sur les corps totalement réels ([Fr-Si]).

On s'intéresse dans cet article au premier cas du théorème de Fermat. Il est bien connu que la présence d'obstructions locales permet de le démontrer sur $\mathbb{Q}$ pour certains exposants $p$. Historiquement, le premier résultat spectaculaire à ce sujet a été obtenu par S. Germain en 1823, qui a démontré que si $2 p+1$ est un nombre premier, le premier cas du théorème de Fermat est vrai sur $\mathbb{Q}$ pour l'exposant $p$. Cet énoncé a été généralisé par de nombreux mathématiciens, notamment par Wendt en 1884 qui a obtenu une généralisation en termes du résultant des polynômes de la forme $X^{n}-1$ et $(X+1)^{n}-1$ (voir [Co-2] p. 430 et $[\mathrm{Ri}]$ p. 137). On se propose ici de démontrer un analogue du critère de Wendt dans le cas où $K$ est un corps quadratique imaginaire. 
Par ailleurs, si pour tout entier $a$ compris entre 1 et $\frac{p-3}{2}$, on a

$$
1+a^{p} \not \equiv(1+a)^{p} \bmod \cdot p^{2}
$$

le premier cas du théorème de Fermat est vrai sur $\mathbb{Q}$ pour l'exposant $p$ ([Co-2] p. 430). Cette condition ne peut être satisfaite que si 3 ne divise pas $p-1$. F.H. Hao et C.J. Parry ont étendu ce critère aux corps quadratiques dont l'anneau d'entiers possède un idéal premier au-dessus de $p$ de degré résiduel 1 ([Ha-Pa]). On obtient ici une généralisation de cet énoncé à d'autres familles de corps de nombres. Pour tout nombre premier $p \geq 5$ vérifiant cette condition modulo $p^{2}$, cela permet par exemple d'établir le premier cas du théorème de Fermat pour $p$, sur les corps cubiques purs, et sur des corps purs de degré sur $\mathbb{Q}$ arbitrairement grand.

J'ai bénéficié de nombreuses remarques de D. Bernardi pendant la rédaction de cet article. Je l'en remercie vivement ici.

\section{I. Énoncé des résultats}

Pour tout corps de nombres $K$, notons $O_{K}$ son anneau d'entiers et $h_{K}$ son nombre de classes. La lettre $p$ désigne un nombre premier impair.

\section{Le critère de Wendt sur les corps quadratiques imaginaires}

Soit $K$ un corps quadratique imaginaire. Pour tout entier $n \geq 1$, notons $W_{n}$ le résultant des polynômes $X^{n}-1$ et $(X+1)^{n}-1$.

Théorème 1. Supposons les conditions suivantes satisfaites :

1) on a $h_{K} \not \equiv 0$ mod. $p$.

2) Il existe $n \geq 1$ tel que $q=n p+1$ soit un nombre premier décomposé dans $K$ et que

$$
\left(n^{n}-1\right) W_{n} \not \equiv 0 \text { mod. } q \text {. }
$$

Alors, le premier cas du théorème de Fermat est vrai sur $K$ pour l'exposant $p$.

Remarque 1. Le nombre premier $p$ étant donné, il n'existe qu'un nombre fini d'entiers $n$ pour lesquels $n p+1$ soit un nombre premier ne divisant pas $W_{n}$. Plus précisément, Dickson a démontré en 1909 que tout nombre premier de la forme $n p+1$, plus grand que $(p-1)^{2}(p-2)^{2}+6 p-2$, divise $W_{n}$ ([Ri] p. 301). De plus, $W_{n}=0$ si 6 divise $n$. La question de savoir si pour tout $p$, il existe $n$ tel que $n p+1$ soit un nombre premier ne divisant pas $W_{n}$ est toujours ouverte. Elle a été posée par Flye Sainte-Marie en 1880 et par Landau en 1913 (loc. cit.). Pour autant, on constate expérimentalement que la seconde condition du théorème est très souvent réalisée en pratique, excepté comme il se doit pour le corps $\mathbb{Q}(\sqrt{-3})$ où elle ne l'est jamais si $p \geq 5$, à cause de la présence des racines cubiques de 
l'unité. Si $K$ n'est pas $\mathbb{Q}(\sqrt{-3})$, il est plausible qu'elle le soit toujours dès que $p$ est plus grand qu'une constante ne dépendant que de $K$.

On déduit de l'égalité $W_{2}=-3$ un analogue du résultat de S. Germain sur $K$.

Corollaire 1. Supposons $h_{K}$ non divisible par $p$ et que $2 p+1$ soit un nombre premier décomposé dans $K$. Alors, le premier cas du théorème de Fermat est vrai sur $K$ pour l'exposant $p$.

Corollaire 2. 1) Si l'on a $p \leq 10^{6}$, le premier cas du théorème de Fermat est vrai sur le corps $\mathbb{Q}(i)$ pour l'exposant $p$.

2) Si $4 p+1$ ou $8 p+1$ ou $16 p+1$ est premier, le premier cas du théorème de Fermat est vrai sur $\mathbb{Q}(i)$ pour l'exposant $p$.

Démonstration : On a $h_{\mathbb{Q}(i)}=1$ et l'on peut vérifier la seconde condition du théorème pour $p \leq 10^{6}$ à l'aide du logiciel de calculs Pari, environ en cinq minutes ([Pari]). Tout nombre premier congru à 1 modulo 4 est décomposé dans $\mathbb{Q}(i)$. Les factorisations de $\left(n^{n}-1\right) W_{n}$ pour $n=4,8,16$, permettent alors d'établir la seconde assertion (cf. loc. cit.).

À titre indicatif, sur le corps $\mathbb{Q}(i)$, la liste des couples $(p, n)$ pour $p<100$, avec les plus petits entiers $n$ pour lesquels le critère fonctionne est la suivante :

$$
\begin{gathered}
(3,4),(5,8),(7,4),(11,8), 13,4),(17,8),(19,40),(23,20),(29,8),(31,76),(37,4),(41,20), \\
(43,4),(47,20),(53,20),(59,20),(61,16),(67,4),(71,8),(73,4),(79,4),
\end{gathered}
$$

$$
(83,32),(89,44),(97,4) \text {. }
$$

\section{Obstructions locales modulo $p^{2}$}

Théorème 2. Soient $K$ un corps de nombres et $p$ un nombre premier impair. Supposons les conditions suivantes satisfaites :

1) il existe un idéal premier de $O_{K}$ au-dessus de $p$, de degré résiduel sur $p$ égal à 1 , et d'indice de ramification sur $p$ inférieur ou égal à $p-1$.

2) On a

$$
1+a^{p} \not \equiv(1+a)^{p} \text { mod. } p^{2} \text { pour tout } a=1,2, \cdots, \frac{p-3}{2} .
$$

Alors, le premier cas du théorème de Fermat est vrai sur $K$ pour l'exposant $p$.

Remarque 2. La condition (1) de l'énoncé ne peut être réalisée que si $p=3$ ou si $p \equiv 2$ mod. 3. En effet, si $p \neq 3$, le polynôme $(X+1)^{p}-X^{p}-1$ est divisible par 
$p\left(X^{2}+X+1\right)$ et les racines cubiques de l'unité appartiennent à $\mathbb{F}_{p}$ si $p \equiv 1 \bmod .3$. L'ensemble des nombres premiers $p<150$ qui la vérifie est

$$
\{3,5,11,17,23,29,41,47,53,71,89,101,107,113,131,137,149\} .
$$

Expérimentalement, on constate qu'environ 84 pour cent des nombres premiers congrus à 2 modulo 3 satisfont la condition (1). Par exemple, il y a 39265 nombres premiers impairs, congrus à 2 modulo 3 , plus petits que $10^{6}$, et 33316 d'entre eux passent positivement le test ; il y en a 30870 qui sont irréguliers et 13192 d'entre eux satisfont la condition (1).

Corollaire 3. Soit $p$ un nombre premier $\geq 5$ vérifiant la condition (1). Soit $d$ un entier rationnel distinct de \pm 1 . Supposons que l'on soit dans l'un des cas suivants :

1) $K=\mathbb{Q}(\sqrt[3]{d})$ où $d$ est sans facteurs cubiques,

2) $K=\mathbb{Q}(\sqrt[n]{d})$ où $p$ ne divise pas $d n$, $d$ est sans facteurs carrés et $n \equiv 1$ mod. $p-1$,

3) $K$ est une extension de $\mathbb{Q}$, totalement ramifiée en $p$, dont le degré sur $\mathbb{Q}$ est inférieur ou égal à $p-1$.

Alors, le premier cas du théorème de Fermat est vrai sur $K$ pour l'exposant $p$.

Remarque 3. Dans l'énoncé de la seconde assertion, l'hypothèse selon laquelle $d$ est distinct de \pm 1 et sans facteurs carrés, sert à garantir que le polynôme $X^{n}-d$ est irréductible

sur $\mathbb{Q}$. Par ailleurs, si l'on spécifie $d$ et $p$, on peut obtenir un énoncé plus précis. À titre indicatif, si $n$ est un entier impair non multiple de 5 , le premier cas du théorème de Fermat est vrai sur le corps $\mathbb{Q}(\sqrt[n]{3})$, pour l'exposant $p=5$.

\section{Démonstration du théorème 1}

Soit $(x, y, z)$ un triplet d'éléments de $O_{K}$ tel que

$$
x^{p}+y^{p}+z^{p}=0 \quad \text { et } \quad(x y z) O_{K}+p O_{K}=O_{K} .
$$

Posons

$$
D=x O_{K}+y O_{K}
$$

On a les égalités

$$
D=x O_{K}+z O_{K}=y O_{K}+z O_{K}
$$

\section{Lemmes préliminaires}

Les égalités (2) et le lemme qui suit n'utilisent pas le fait que $K$ est un corps quadratique imaginaire. 


\section{Lemme 1. L'idéal}

$$
\frac{(x+y) O_{K}}{D}
$$

est la puissance $p$-ième d'un idéal de $O_{K}$.

Démonstration : On a l'égalité

$$
(x+y) s=-z^{p} \quad \text { où } \quad s=\sum_{k=0}^{p-1} x^{p-1-k}(-y)^{k} .
$$

Parce que $x$ et $y$ sont dans $D$, l'idéal $D^{p-1}$ divise $s O_{K}$. On obtient l'égalité d'idéaux de $O_{K}$

$$
\left(\frac{(x+y) O_{K}}{D}\right)\left(\frac{s O_{K}}{D^{p-1}}\right)=\left(\frac{z O_{K}}{D}\right)^{p} .
$$

Il suffit ainsi d'établir que l'on a

$$
\frac{(x+y) O_{K}}{D}+\frac{s O_{K}}{D^{p-1}}=O_{K}
$$

Soit $\mathfrak{q}$ un idéal premier non nul de $O_{K}$ divisant $\frac{(x+y) O_{K}}{D}$. Il s'agit de montrer que $\mathfrak{q}$ ne divise pas $\frac{s O_{K}}{D^{p-1}}$. Pour cela, on vérifie par récurrence que pour tout $k \geq 1$, on a

$$
(-y)^{k} \equiv x^{k} \text { mod. } \mathfrak{q} D^{k}
$$

Pour tout $k$ compris entre 0 et $p-1$, on a donc

$$
x^{p-1-k}(-y)^{k} \equiv x^{p-1} \bmod \cdot \mathfrak{q} D^{p-1},
$$

d'où la congruence

$$
s \equiv p x^{p-1} \bmod . \mathfrak{q} D^{p-1} .
$$

Supposons que $\mathfrak{q}$ divise $\frac{s O_{K}}{D^{p-1}}$. L'idéal $\mathfrak{q} D^{p-1}$ divise alors $\left(p x^{p-1}\right) O_{K}$. Par ailleurs, l'égalité $(x y z) O_{K}+p O_{K}=O_{K}$ entraîne que $\mathfrak{q} D^{p-1}$ est premier avec $p O_{K}$, donc $\mathfrak{q} D^{p-1}$ divise $x^{p-1} O_{K}$. On en déduit que $\mathfrak{q}$ divise $\frac{x O_{K}}{D}$, puis que $x$ est dans $\mathfrak{q} D$. L'élément $x+y$ étant aussi dans $\mathfrak{q} D$, il en est de même de $y$. Cela contredit le fait que $D$ est le plus grand commun diviseur de $x O_{K}$ et $y O_{K}$, d'où le lemme.

Le fait que $K$ soit un corps quadratique imaginaire intervient désormais de façon essentielle. On supposera de plus, ce qui n'est pas restrictif,

$$
K \neq \mathbb{Q}(\sqrt{-3})
$$

En effet, on a $h_{\mathbb{Q}(\sqrt{-3})}=1$, la seconde condition du théorème 1 est satisfaite pour $p=3$ (avec $n=2$ ) et ne l'est pas si $p \geq 5$. Par ailleurs, il est connu que le premier cas du 
théorème de Fermat est vrai sur $\mathbb{Q}(\sqrt{-3})$ pour l'exposant $p=3$. Le théorème 1 est donc vrai pour le corps $\mathbb{Q}(\sqrt{-3})$.

Par hypothèse, $p$ ne divise pas $h_{K}$. Il existe donc $t \in \mathbb{N}$ tel que $p$ divise $t h_{K}+1$. L'idéal $D^{h_{K}}$ est principal. En particulier, il existe $d \in O_{K}$ tel que l'on ait

$$
D^{h_{K} t}=d O_{K}
$$

Lemme 2. Il existe des éléments non nuls $a, b, c$ dans $O_{K}$ tels que l'on ait

$$
d(x+y)=a^{p}, \quad d(x+z)=b^{p}, \quad d(y+z)=c^{p} .
$$

Démonstration : D'après le lemme 1, il existe un idéal $I$ de $O_{K}$ tel que l'on ait l'égalité $(x+y) O_{K}=D I^{p}$. On a donc

$$
\left(D^{\frac{h_{K} t+1}{p}} I\right)^{p}=d(x+y) O_{K}
$$

Parce que $p$ ne divise pas $h_{K}$, l'idéal $D^{\frac{h_{K} t+1}{p}} I$ est principal. Le corps $K$ étant quadratique imaginaire, distinct de $\mathbb{Q}(\sqrt{-3})$, les unités de $O_{K}$ sont des puissances $p$-ièmes dans $O_{K}$ (y compris si $p=3$ ), d'où l'existence d'un élément $a \in O_{K}$ tel que $d(x+y)=a^{p}$. Les égalités (2) et (3) entraînent alors le résultat.

Soit $n \geq 1$ un entier tel que $q=n p+1$ soit un nombre premier vérifiant la seconde condition de l'énoncé du théorème.

Lemme 3. Chaque idéal premier de $O_{K}$ au-dessus de $q$ divise $(x y z) O_{K}$.

Démonstration: Supposons qu'il existe un idéal premier $\mathfrak{q}$ de $O_{K}$ au-dessus de $q$ ne divisant pas $(x y z) O_{K}$. Il existe alors $u \in O_{K}$ tel que l'on ait

$$
u \equiv\left(\frac{x}{z}\right)^{\frac{q-1}{n}} \bmod \cdot \mathfrak{q}
$$

Le corps $O_{K} / \mathfrak{q}$ est de cardinal $q$, d'où la congruence

$$
u^{n}=1 \bmod \cdot \mathfrak{q}
$$

L'égalité $x^{p}+y^{p}+z^{p}=0$ implique

$$
u+1 \equiv-\left(\frac{y}{z}\right)^{\frac{q-1}{n}} \bmod . \mathfrak{q} .
$$

On obtient ( $n$ est pair)

$$
(u+1)^{n} \equiv 1 \bmod \cdot \mathfrak{q},
$$


ce qui entraîne que $q$ divise $W_{n}$, d'où une contradiction et le résultat.

\section{Fin de la démonstration du théorème 1}

Quitte à diviser l'égalité $x^{p}+y^{p}+z^{p}=0$ par une puissance convenable de $q$, on peut supposer que $(x, y, z)$ n'est pas nul modulo $q O_{K}$. Il existe donc un idéal premier $\mathfrak{q}$ de $O_{K}$ au-dessus de $q$ tel que $(x, y, z)$ soit non nul modulo $\mathfrak{q}$. Notons $v_{\mathfrak{q}}$ la valuation sur $K$ qui lui est associée. D'après le lemme 3 , on peut supposer que l'on a

$$
v_{\mathfrak{q}}(z) \geq 1,
$$

auquel cas on a

$$
v_{\mathfrak{q}}(x)=v_{\mathfrak{q}}(y)=0 .
$$

En particulier, $\mathfrak{q}$ ne divise pas $D$ et d'après l'égalité (3), on a

$$
v_{\mathfrak{q}}(d)=0 .
$$

Par suite, on a $v_{\mathfrak{q}}(d(y+z))=0$ et d'après le lemme 2 on obtient

$$
v_{\mathfrak{q}}(c)=0 .
$$

Pour la même raison, on a

$$
v_{\mathfrak{q}}(b)=0 .
$$

L'égalité $2 d z=b^{p}+c^{p}-a^{p}$ (lemme 2) et l'hypothèse selon laquelle $q$ ne divise pas $W_{n}$, impliquent alors (comme dans la démonstration du lemme 3)

$$
v_{\mathfrak{q}}(a) \geq 1 .
$$

Il en résulte que l'on a

$$
v_{\mathfrak{q}}(x+y) \geq 1 .
$$

On a ainsi l'égalité

$$
d\left(-z^{p}\right)=a^{p} s \quad \text { avec } \quad s=\sum_{k=0}^{p-1} x^{p-1-k}(-y)^{k},
$$

et la congruence

$$
s \equiv p x^{p-1} \bmod . \mathfrak{q} .
$$

On en déduit que $v_{\mathfrak{q}}(s)=0$, donc $\frac{z}{a}$ est une unité modulo $\mathfrak{q}$. On a $d x \equiv b^{p}$ mod. $\mathfrak{q}$, d'où

$$
p \equiv \frac{s}{x^{p-1}} \equiv\left(\frac{-b z}{x a}\right)^{p} \text { mod. } \mathfrak{q} .
$$


Le corps $O_{K} / \mathfrak{q}$ est de cardinal $q$ et on a $n p=q-1$, d'où $p^{n} \equiv 1$ mod. $\mathfrak{q}$, puis

$$
p^{n} \equiv 1 \text { mod. } q \text {. }
$$

Puisque $n$ est pair, on obtient

$$
1=(-1)^{n}=(n p-q)^{n} \equiv n^{n} p^{n} \equiv n^{n} \text { mod. } q,
$$

ce qui conduit à une contradiction, d'où le théorème.

\section{Démonstration du théorème 2}

Elle est analogue à celle du théorème 1 de $[\mathrm{Ha}-\mathrm{Pa}]$. Soit $\mathfrak{p}$ un idéal premier de $O_{K}$ au-dessus de $p$ de degré résiduel 1 . Notons $v_{\mathfrak{p}}$ la valuation sur $K$ qui lui est associée. Posons $e=v_{\mathfrak{p}}(p)$ l'indice de ramification de $\mathfrak{p}$ sur $p$. Supposons qu'il existe $x, y, z$ dans $O_{K}$ tels que l'on ait

$$
x^{p}+y^{p}+z^{p}=0 \quad \text { et } \quad v_{\mathfrak{p}}(x y z)=0 .
$$

Les corps $O_{K} / \mathfrak{p}$ et $\mathbb{F}_{p}$ étant isomorphes, il existe $x_{0}, y_{0}, z_{0} \in \mathbb{Z}$ non divisibles par $p$ tels que

$$
x \equiv x_{0} \bmod . \mathfrak{p}, y \equiv y_{0} \bmod . \mathfrak{p}, z \equiv z_{0} \bmod . \mathfrak{p} .
$$

Il en résulte que l'on a

$$
v_{\mathfrak{p}}\left(x^{p}-x_{0}^{p}\right) \geq \operatorname{Inf}(e+1, p)=e+1,
$$

d'où la congruence

$$
x_{0}^{p}+y_{0}^{p}+z_{0}^{p} \equiv 0 \bmod . \mathfrak{p}^{e+1} .
$$

On en déduit que l'on a

$$
x_{0}^{p}+y_{0}^{p}+z_{0}^{p} \equiv 0 \text { mod. } p^{2} .
$$

En particulier, on a

$$
x_{0}+y_{0}+z_{0} \equiv 0 \text { mod. } p,
$$

d'où

$$
\left(x_{0}+y_{0}\right)^{p}+z_{0}^{p} \equiv 0 \text { mod. } p^{2},
$$

puis

$$
\left(x_{0}+y_{0}\right)^{p} \equiv x_{0}^{p}+y_{0}^{p} \text { mod. } p^{2} .
$$

On obtient

$$
1+a^{p} \equiv(1+a)^{p} \text { mod. } p^{2} \quad \text { avec } \quad a \equiv x_{0}^{-1} y_{0} \bmod . p^{2} .
$$

On a $a \not \equiv 0$ mod. $p$ et $a \not \equiv-1$ mod. $p$ car $p$ ne divise pas $z_{0}$. Parce que (4) ne dépend que de la congruence de $a$ modulo $p$, on peut supposer que l'on a $1 \leq a \leq p-2$. Si $a=\frac{p-1}{2}$, alors $a=1$ est aussi solution de (4). Si l'on a $\frac{p-1}{2}<a \leq p-2$, alors $p-1-a$ satisfait (4) et $1 \leq p-1-a \leq \frac{p-3}{2}$. Cela contredit la condition (1), d'où le résultat. 


\section{Démonstration du corollaire 3}

1) On a $p \equiv 2 \bmod .3$ car $p$ satisfait la condition (1). Dans l'anneau d'entiers de $\mathbb{Q}(\sqrt[3]{d})$, il existe donc un idéal premier au-dessus de $p$ de degré résiduel 1 ([Co-1], cor. 6.4.15 et th. 6.4.16), d'où la première assertion.

2) Posons $K=\mathbb{Q}(\sqrt[n]{d})$. Le polynôme $X^{n}-d$ est irréductible sur $\mathbb{Q}$, de discriminant

$$
(-1)^{\frac{n(n-1)}{2}} n^{n} d^{n-1} \text {. }
$$

La congruence $n \equiv 1 \bmod . p-1$ implique $d^{n} \equiv d \bmod$. $p$. Le fait que $p$ ne divise pas $d n$ entraîne alors l'existence d'un idéal premier de $O_{K}$ au-dessus de $p$ non ramifié de degré résiduel 1 ([Co-1], th. 4.8.13), d'où le résultat.

3) La dernière assertion est une conséquence directe du théorème 2.

\section{Bibliographie}

[Co-1] H. Cohen, A course in Computational Algebraic Number Theory, Springer-Verlag GTM 138, 1993.

[Co-2] H. Cohen, Number Theory Volume I : Tools and Diophantine Equations, SpringerVerlag GTM 239, 2007.

[Fr-Si] N. Freitas et S. Siksek, Modularity and the Fermat equation over totally real number fields, arXiv : 1307.3162v2 (2014), 32 pages.

[Ha-Pa] F. H. Hao, C. J. Parry, The Fermat equation over quadratic fields, J. Number Theory 19 (1984), 115-130.

[Ja-Me] F. Jarvis et P. Meekin, The Fermat equation over $\mathbb{Q}(\sqrt{2})$, J. Number Theory 109 (2004), 182-196.

[Pari] C. Batut, D. Bernardi, K. Belabas, H. Cohen et M. Olivier, PARI-GP, version 2.3.3, Université de Bordeaux I, (2008).

[Ri] P. Ribenboim, Fermat's Last Theorem for Amateurs, Springer-Verlag, 1999.

[Wi] A. Wiles, Modular elliptic curves and Fermat's Last Theorem, Ann. of Math. 141 (1995), 443-551.

\footnotetext{
Alain Kraus

Université de Paris VI,

Institut de Mathématiques,

4 Place Jussieu, 75005 Paris,

France

e-mail : alain.kraus@imj-prg.fr
} 